\title{
A Novel Role of Chloride Channels in Cerebellar Ataxia
}

\author{
Friederike Auer*
}

'Department of Animal Physiology, Centre for Organismal Studies, University of Heidelberg, Heidelberg, Germany

\section{Article Info}

\section{Article Notes}

Received: July 18, 2018

Accepted: August 14, 2018

\section{${ }^{*}$ Correspondence}

Ms. Friederike Auer, Department of Animal Molecular

Physiology, Centre for Organismal Studies, Heidelberg

University, Im Neuenheimer Feld 504, 69120 Heidelberg,

Germany; Telephone No: +49(0)6221 54 5664;

E-mail: friederike.auer@cos.uni-heidelberg.de.

C 2018 Auer F. This article is distributed under the terms of the Creative Commons Attribution 4.0 International License

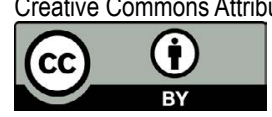

\section{Keywords}

Cerebellum

Ataxia

Anoctamin

TMEM16B

Chloride

Purkinje cell
Abstract

Cerebellar ataxias are characterized by a disturbance of motor coordination and learning. To understand the molecular mechanisms underlying especially hereditary cerebellar ataxias, many mouse models, often with altered Purkinje cell firing, have been studied in the past decades. Of particular interest were mouse lines with mutations in ion channels or their ligands. In this context, a recently described $\mathrm{ANO}^{--}$mouse line has been reported to show a variety of ataxic symptoms, ranging from gait abnormalities and problems in motor coordination to severely impaired motor learning. These mice lack the calcium-gated chloride channel Anoctamin 2 (ANO2). First studies, focusing on the molecular mechanisms underlying the ataxic phenotype of $\mathrm{ANO}^{--}$mice, indicate an involvement of ANO2 in two different processes in the neuronal network of the cerebellar cortex. Both mechanisms are thought to modulate the firing pattern of cerebellar Purkinje cells and could, therefore, explain the ataxic phenotype of $\mathrm{ANO}^{-\%}$ mice. This review summarizes the so far obtained data regarding the role of ANO2 in the coordination and learning of movements.

\section{Introduction}

The cerebellum plays an important role in the control, coordination, and learning of movements. Malfunctions in these processes are referred to as cerebellar ataxia, which can have multiple causes. These are divided into acquired and hereditary ataxias, which can be autosomal recessive like the Friedreich's ataxia ${ }^{1}$ or autosomal dominant like different types of spinocerebellar ataxias ${ }^{2}$. Among the non-hereditary ataxias the most prevalent cause of cerebellar dysfunction is multiple sclerosis ${ }^{3}$. Other reasons can be lesions of the cerebellum, stroke as well as various drugs (e.g. ethanol ${ }^{4}$, benzodiazepines, antiepileptic drugs and others ${ }^{5}$ ).

Patients suffering from cerebellar ataxia have problems in performing smooth, well-coordinated, target-directed movements. Typical signs are impairments in coordination of limb movements, especially in multijoint movements ${ }^{6}$, referred to as dyssynergia, as well as in performing rapidly alternating movements (dysdiadochokinesis). Other symptoms are dysmetria, which is defined as over- or undershooting while reaching a target, and the occurrence of action tremor. The tremor amplitude increases when approaching a target, a phenomenon referred to as intention tremor ${ }^{7}$. Besides voluntary planned limb movements, also gait and balance in general, are often affected, resulting in inaccurate foot placement and increased variability in stride length ${ }^{8}$. Problems in oculomotor control and speech production (dysarthria) can also occur. In addition to these impairments in the coordination of muscles, dysfunctions in the cerebellum can also lead to problems in motor learning of both simple and complex motor skills, especially in adaptation and error-based motor learning tasks 9 . This affects the everyday life of the patients as they have problems whenever fast adaptation is required, 
for example after putting on glasses or taking an object that serves as an extension of the arm like a pen or silverware.

To examine the molecular mechanisms underlying hereditary cerebellar ataxias, several animal models of ataxia are available. Many of them show Purkinje cell (PC) dysfunction and/or degeneration. The PCs are the sole output of the cerebellum and a wide variety of mutations affecting either upstream pathways or the PC itself will alter its firing pattern or frequency ${ }^{10}$. The PCs receive excitatory input via parallel fibers and climbing fibers which arise in the inferior olivary nucleus of the brainstem. These inputs shape the spontaneous firing pattern of the PCs which is further modulated by a network of inhibitory interneurons located in the molecular layer of the cerebellar cortex (MLIs) (Figure 1).

Hoxha et al. $2018^{10}$ summarize mouse models with ataxic symptoms that have mutations in sodium, potassium or calcium channels or their ligands. In the past years another mouse model that is lacking an ion channel in the olivocerebellar system and is showing ataxic symptoms has been studied: the Anoctamin 2 (ANO2) KO mouse $\left(\mathrm{ANO}^{\%} /\right)^{11,12}$.

\section{Ataxia in $\mathrm{ANO}^{-/-}$Mice}

As mentioned above, gait abnormalities are a common sign of cerebellar ataxia in human patients. In mice models, this feature is usually tested via footprint analysis ${ }^{13}$ or fully automated systems like CatWalk XT analysis apparatus ${ }^{14,15}$.
Although ANO2\% mice displayed normal basal voluntary activities, the animals showed a slow, hesitant and irregular gait with compromised interpaw coordination in CatWalk analysis $^{11}$.

This first hint towards an ataxic phenotype of ANO2\% was confirmed by performing the horizontal ladder test, where the mice had to cross a series of rungs with randomly set gaps. Here ANO2\% mice showed no obvious impairment in limb placement but nevertheless needed more time and steps to cross the ladder, thus appearing insecure and cautious in this task. As motor performance was impaired in general, running wheel experiments were performed. ANO2 $\%$ mice spent the same time running on the wheel as wildtypes, indicating similar motivation, but reached $70 \%$ less rotations (Figure 2a). They also showed almost no improvement over several nights of training while wildtype mice doubled their performance. These observations suggested that, besides gait and motor performance, motor learning was also impaired in ANO2\% mice ${ }^{11}$. To address this issue, the accelerating rotarod test was performed, which is currently the most commonly used test to examine motor coordination and learning in mice. The mice are placed on a beam that rotates at accelerating speed and the latency to fall off the rod is measured. Interestingly, ANO2 $\%$ mice and wildtype mice performed similarly well on the rotarod on the first day of testing ${ }^{11}$. As in the running wheel experiments, ANO2\% mice showed only a small improvement over six days of testing in contrast to the

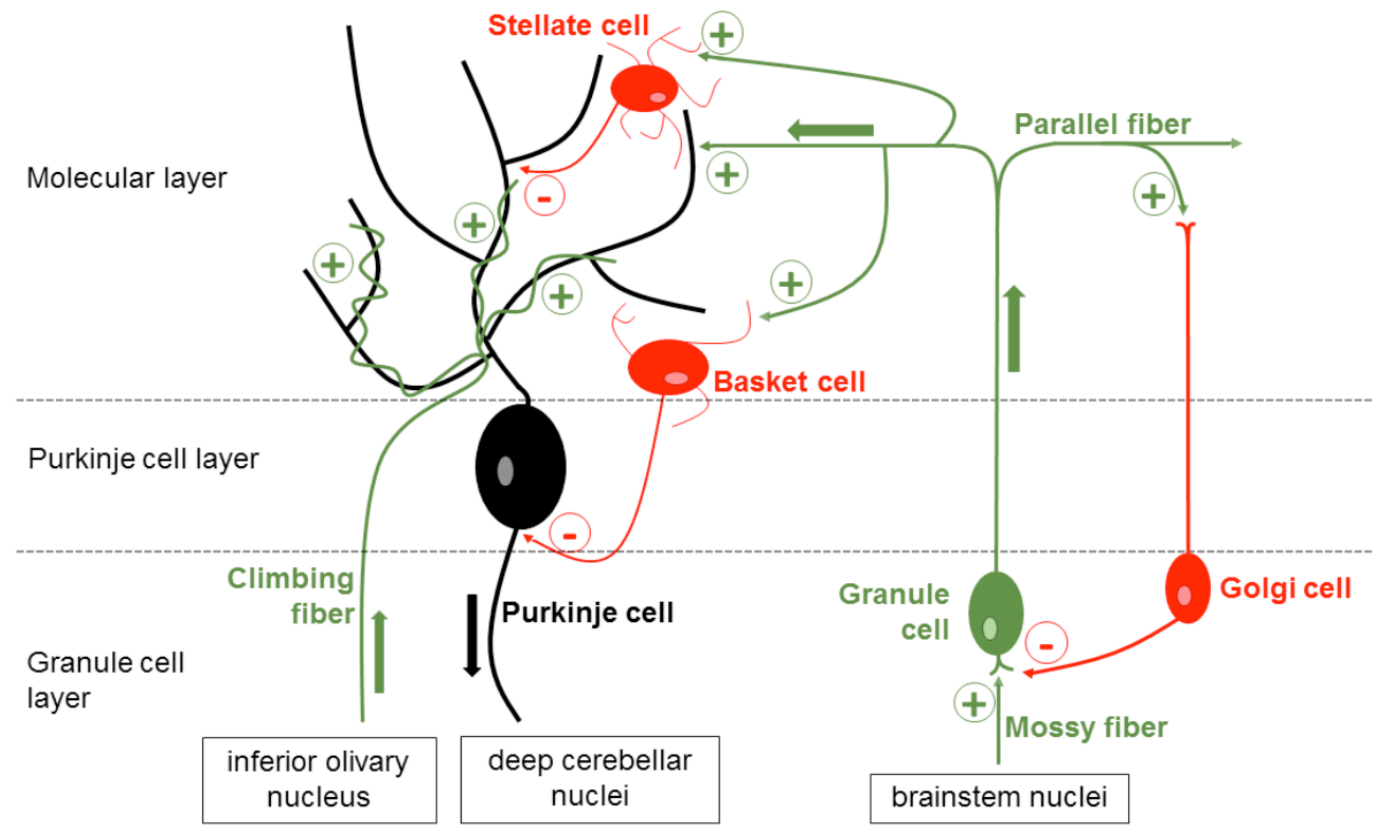

Figure 1: Microcircuitry of the cerebellum

Schematic illustration of the neuronal network in the cerebellar cortex and the afferent and efferent pathways. Purkinje cells (black) receive excitatory input via parallel fibers and climbing fibers (green) and inhibitory input by stellate and basket cells (red). The Purkinje cells project to the deep cerebellar nuclei from where the information is distributed to various targets outside the cerebellum. 
a

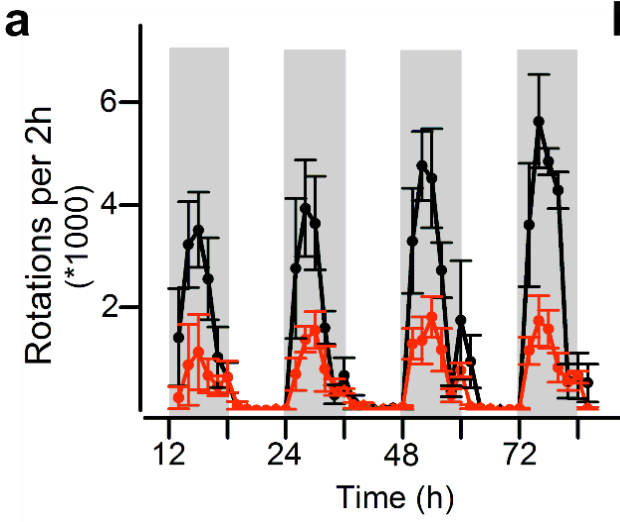

b

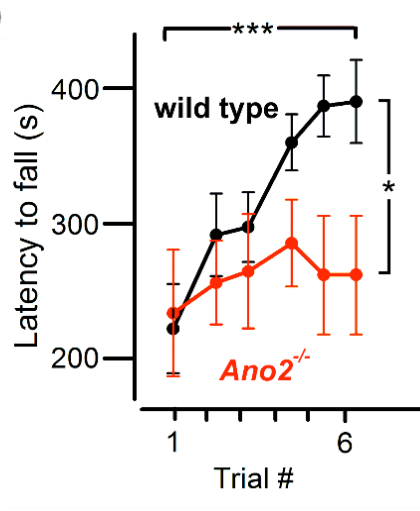

C

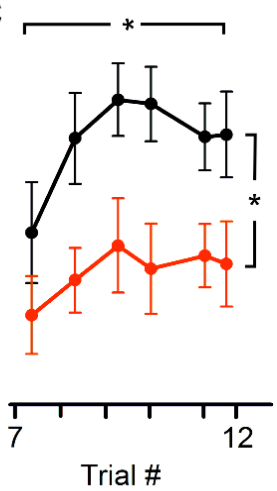

Figure 2: Impaired motor performance and learning in ANO2 $\%$ mice

(from Neureither et al., 2017 $7^{11}$; http:// creativecommons.org/licenses/by/4.0/)

(a) Results from running wheel experiments. Wildtype mice (black) reached twice as many rotations as ANO2 $\%$ mice (red) and also improved their performance over four consecutive nights. In contrast, $\mathrm{ANO}^{-/}$mice were not able to improve their performance, pointing towards an impairment of motor coordination and learning in ANO2 ${ }^{-1}$ mice. (b), (c) Results from accelerating rotarod experiments. (b) Wildtype (black) and $\mathrm{ANO}^{-}$- mice (red) performed similarly on the first day of testing, but only wildtypes were able to improve their performance significantly. (c) After a break of 20 days wildtype mice (black) showed their maximum performance already on the second day of testing, whereas $\mathrm{ANO}^{-\%}$ mice (red) showed no significant improvement compared to the initial testing period. This indicates that the consolidated procedural memory is impaired in $\mathrm{ANO}^{-\%}$ mice.

wildtypes that almost doubled their performance (Figure 2b). To examine whether the consolidation of procedural memory is affected as well, the mice were tested on the rotarod again after a break of three weeks. ANO2\% mice displayed the same low performance throughout the testing period as they did in the initial trials, whereas wildtype mice reached their maximal performance on the second day of testing (Figure 2c). Procedural learning impairment was also seen in a different ANO2\% line through classical eyeblink conditioning ${ }^{12}$, a well-established cerebellardependent learning task ${ }^{16}$. In this test, a sound and a light signal (conditioned stimulus) are given to announce the arrival of an air puff (unconditioned stimulus). ANO2\% mice showed a significant delay in developing conditioned responses compared to wildtype mice which also indicates impairments in cerebellar motor learning ${ }^{12}$.

The published behavioral analyses performed on $\mathrm{ANO}^{-} \%$ mice ${ }^{11,12}$ are commonly used to assess ataxia in mouse models ${ }^{17-19}$. They are suitable to examine several features of ataxia including gait abnormalities, coordination problems, and impaired motor learning.

\section{Reasons for Ataxia in ANO2 ${ }^{-/-}$Mice}

ANO2 is a calcium-gated chloride channel belonging to the protein family of anoctamins and is exclusively expressed in neurons. It was first described in olfactory sensory neurons in the olfactory epithelium ${ }^{20}$ where it plays a role in the detection of weak, unknown odor stimuli ${ }^{21}$. It is also expressed in the glomeruli of the olfactory bulb as well as in the retina ${ }^{22}$, the hippocampus ${ }^{23}$, the striatum ${ }^{24}$ and thalamocortical neurons ${ }^{25}$. As ANO2\% mice displayed problems in cerebellar-dependent motor coordination and learning tasks, expression of ANO2 in the olivocerebellar system seems to be plausible. And, indeed, first evidence is pointing towards an expression in inferior olivary climbing fibers ${ }^{12}$ and $\mathrm{PCs}^{26}$. First results have been obtained that may help to understand the precise role of ANO2 in these two cell types.

MLI synapses are modulated by a mechanism of short-term ionic plasticity termed depolarizationinduced depression of inhibition (DDI) ${ }^{26,27}$. In DDI, ANO2 is supposed to be responsible for transiently diminishing the chloride-gradient across the dendritic membrane at MLI-PC synapses after depolarization through climbing fiber activity ${ }^{26}$. This leads to a reduction in the amplitude of inhibitory postsynaptic currents (IPSCs). In ANO2\% mice such dampening of the inhibitory input on Purkinje cells after climbing fiber activation is absent ${ }^{26}$.

A relationship between ataxia and alterations at MLI-PC synaptic strength has previously been reported. The longknown 'ducky' mutant that is characterized by an ataxic gait ${ }^{28}$ has been shown to have an increased amplitude in spontaneous IPSCs ${ }^{29}$. This mouse line lacks the auxiliary $\mathrm{Ca}^{2+}$ channel subunit $\alpha 2 \delta-2$ which leads to an impaired signaling via the cannabinoid receptor $\mathrm{CB}_{1}$. This receptor plays an important role in another type of short-term plasticity at MLI-PC synapses, called depolarization-induced suppression of inhibition (DSI) ${ }^{30}$. Another mouse line that lacks the plasma membrane $\mathrm{Ca}^{2+}$-ATPase 2 displays severe ataxia $^{31}$. A few years ago it was revealed that these mice also have an increased amplitude as well as an increased frequency of spontaneous IPSCs ${ }^{32}$. Taken together, these data suggest that a disturbed firing pattern of PCs, resulting from an altered input received from MLI, may cause ataxia. 
In inferior olivary neurons, ANO2 is necessary for rapid repolarization after action potential firing ${ }^{12}$. ANO2 $\%$ mice showed a prolonged after-hyperpolarization potential (AHP) and a large after-depolarization potential (ADP). These findings indicate that ANO2 plays an important role in controlling the excitability of climbing fibers. Climbing fibers in ANO2 $\%$ mice may elicit fewer complex spikes in PCs and thus initiate weaker long-term depression (LTD) ${ }^{12}$. To test this hypothesis, it would be interesting to see the effects on PC firing in behaving animals.

The link between climbing fiber innervation of PCs and ataxia, especially some types of spinocerebellar ataxia, has been shown before ${ }^{10,33}$. In most cases, these are developmental defects leading to an innervation of PCs by multiple climbing fibers instead of just one. But not only the architecture of climbing fiber innervation can lead to ataxia. Also, changes in climbing fiber signaling have been shown to cause ataxia, as the loss of a potassium channel in PCs causes silencing of climbing fiber-evoked complex spike activity $^{34}$.

Both mechanisms described above are thought to modulate the output of the cerebellum by shaping the firing pattern of PCs, a common characteristic of ataxic mouse models. Whether the observed ataxic phenotype in ANO2 $\%$ mice relies on an altered input on PCs by MLIs or climbing fibers has to be examined in the future when conditional knockout mice for ANO2 in climbing fibers and Purkinje cells become available ${ }^{35}$.

As stated above, ANO2 is also expressed in other parts of the central nervous system and this could certainly contribute to the observed phenotype in the global $\mathrm{ANO}^{\%}$ mutants. Especially in the striatum, where incoming signals are filtered, ANO2 is expressed in low-threshold spiking, NPY-positive interneurons ${ }^{24}$. Dysfunctions in microcircuits of the striatum have shown to be involved in movement disorders $^{36}$. For now, the results obtained with the global ANO2 $\%$ mice are consistent in showing mild ataxia and impaired motor learning in well-established cerebellardependent tasks. The behavioral data can be linked to functional deficits in inferior olivary climbing fibers and PCs.

\section{Conclusion}

ANO2 $\%$ mice show signs of mild ataxia and a reduced motor learning ability indicating an important role of ANO2 in the coordination of movements and motor learning in the cerebellum. Currently, the molecular mechanisms underlying this phenotype are not entirely understood as ANO2 seems to be involved in more than one component of signal processing in the cerebellar cortex. In future research, the use of conditional ANO2 knockouts as well as in vivo studies during the performance of motor tasks will help to understand the precise role of ANO2 in motor coordination and learning.

\section{Acknowledgement}

This work was supported through a grant by the Evangelische Studienwerk Villigst.

\section{References}

1. Campuzano V, Montermini L, Molto MD, et al. Friedreich's ataxia: autosomal recessive disease caused by an intronic GAA triplet repeat expansion. Science (New York, NY). 1996; 271: 1423-1427.

2. Klockgether T. The clinical diagnosis of autosomal dominant spinocerebellar ataxias. Cerebellum (London, England). 2008; 7: 101105.

3. Kis B, Rumberg B, Berlit P. Clinical characteristics of patients with lateonset multiple sclerosis. Journal of neurology. 2008; 255: 697-702.

4. Dar MS. Ethanol-Induced Cerebellar Ataxia: Cellular and Molecular Mechanisms. Cerebellum (London, England). 2015; 14: 447-465.

5. van Gaalen J, Kerstens FG, Maas RP, et al. Drug-induced cerebellar ataxia: a systematic review. CNS drugs. 2014; 28: 1139-1153.

6. Bastian AJ, Zackowski KM, Thach WT. Cerebellar ataxia: torque deficiency or torque mismatch between joints. Journal of neurophysiology. 2000; 83: 3019-3030.

7. Deuschl G, Bain P, Brin M. Consensus statement of the Movement Disorder Society on Tremor. Ad Hoc Scientific Committee. Movement disorders : official journal of the Movement Disorder Society. 1998; 13 Suppl 3: 2-23.

8. Stolze H, Klebe S, Petersen G, et al. Typical features of cerebellar ataxic gait. Journal of neurology neurosurgery and psychiatry. 2002; 73: 310-312.

9. Doyon J, Benali H. Reorganization and plasticity in the adult brain during learning of motor skills. Current opinion in neurobiology. 2005; $15: 161-167$

10. Hoxha E, Balbo I, Miniaci MC, et al. Purkinje Cell Signaling Deficits in Animal Models of Ataxia. Frontiers in synaptic neuroscience. 2018; 10: 6 .

11. Neureither F, Ziegler K, Pitzer C, et al. Impaired Motor Coordination and Learning in Mice Lacking Anoctamin 2 Calcium-Gated Chloride Channels. Cerebellum (London, England). 2017.

12. Zhang Y, Zhang Z, Xiao S, et al. Inferior Olivary TMEM16B Mediates Cerebellar Motor Learning. Neuron. 2017; 95: 1103-1111.e1104.

13. Clark HB, Burright EN, Yunis WS, et al. Purkinje cell expression of a mutant allele of SCA1 in transgenic mice leads to disparate effects on motor behaviors, followed by a progressive cerebellar dysfunction and histological alterations. The Journal of neuroscience the official journal of the Society for Neuroscience. 1997; 17: 7385-7395.

14. Hamers FP, Koopmans GC, Joosten EA. CatWalk-assisted gait analysis in the assessment of spinal cord injury. Journal of neurotrauma. 2006; 23: 537-548.

15. Cendelin J, Voller J, Vozeh F. Ataxic gait analysis in a mouse model of the olivocerebellar degeneration. Behavioural brain research. 2010; 210: 8-15.

16. Heiney SA, Wohl MP, Chettih SN, et al. Cerebellar-dependent expression of motor learning during eyeblink conditioning in headfixed mice. The Journal of neuroscience the official journal of the Society for Neuroscience. 2014; 34: 14845-14853.

17. Jayabal S, Ljungberg L, Erwes T, et al. Rapid Onset of Motor Deficits in a Mouse Model of Spinocerebellar Ataxia Type 6 Precedes Late Cerebellar Degeneration. eNeuro. 2015; 2.

18. Wang JY, Yu IS, Huang CC, et al. Sun1 deficiency leads to cerebellar ataxia in mice. Disease models \& mechanisms. 2015; 8: 957-967. 
19. Brooks SP, Dunnett SB. Tests to assess motor phenotype in mice: user's guide. Nature reviews Neuroscience. 2009; 10: 519-529.

20. Stephan AB, Shum EY, Hirsh S, et al. ANO2 is the cilial calciumactivated chloride channel that may mediate olfactory amplification. Proceedings of the National Academy of Sciences of the United States of America. 2009; 106: 11776-11781.

21. Neureither F, Stowasser N, Frings $S$, et al. Tracking of unfamiliar odors is facilitated by signal amplification through anoctamin 2 chloride channels in mouse olfactory receptor neurons. Physiological reports. $2017 ; 5$.

22. Stohr H, Heisig JB, Benz PM, et al. TMEM16B, a novel protein with calcium-dependent chloride channel activity, associates with a presynaptic protein complex in photoreceptor terminals. The Journal of neuroscience : the official journal of the Society for Neuroscience. 2009; 29: 6809-6818.

23. Huang WC, Xiao S, Huang F, et al. Calcium-activated chloride channels (CaCCs) regulate action potential and synaptic response in hippocampal neurons. Neuron. 2012; 74: 179-192.

24. Song SC, Beatty JA, Wilson CJ. The ionic mechanism of membrane potential oscillations and membrane resonance in striatal LTS interneurons. Journal of neurophysiology. 2016; 116: 1752-1764.

25. Ha GE, Lee J, Kwak H, et al. The Ca(2+)-activated chloride channel anoctamin-2 mediates spike-frequency adaptation and regulates sensory transmission in thalamocortical neurons. Nature communications. 2016; 7: 13791 .

26. Zhang W, Schmelzeisen S, Parthier D, et al. Anoctamin CalciumActivated Chloride Channels May Modulate Inhibitory Transmission in the Cerebellar Cortex. PloS one. 2015; 10: e0142160.

27. Satoh H, Qu L, Suzuki H, et al. Depolarization-induced depression of inhibitory transmission in cerebellar Purkinje cells. Physiological reports. 2013; 1: e00061.
28. Snell GD. DUCKY, A NEW SECOND CHROMOSOME MUTATION IN THE MOUSE. Journal of Heredity. 1955; 46: 27-29.

29. Wang X, Whalley BJ, Stephens GJ. The du(2J) mouse model of ataxia and absence epilepsy has deficient cannabinoid $\mathrm{CB}(1)$ receptormediated signalling. The Journal of physiology. 2013; 591:3919-3933.

30. Kreitzer AC, Regehr WG. Cerebellar depolarization-induced suppression of inhibition is mediated by endogenous cannabinoids. The Journal of neuroscience the official journal of the Society for Neuroscience. 2001; 21: Rc174.

31. Kozel PJ, Friedman RA, Erway LC, et al. Balance and hearing deficits in mice with a null mutation in the gene encoding plasma membrane Ca2+-ATPase isoform 2. The Journal of biological chemistry. 1998; 273: 18693-18696.

32. Empson RM, Huang H, Nagaraja RY, et al. Enhanced synaptic inhibition in the cerebellar cortex of the ataxic PMCA2(-/-) knockout mouse. Cerebellum (London, England). 2013; 12: 667-675.

33. Smeets CJ, Verbeek DS. Climbing fibers in spinocerebellar ataxia: A mechanism for the loss of motor control. Neurobiology of disease. 2016; 88: 96-106.

34. Chen X, Kovalchuk Y, Adelsberger H, et al. Disruption of the olivocerebellar circuit by Purkinje neuron-specific ablation of BK channels. Proceedings of the National Academy of Sciences of the United States of America. 2010; 107: 12323-12328.

35. Slugocka A, Wiaderkiewicz J, Barski JJ. Genetic Targeting in Cerebellar Purkinje Cells: an Update. Cerebellum (London, England). 2017; 16: 191-202.

36. Gittis AH, Kreitzer AC. Striatal microcircuitry and movement disorders. Trends in neurosciences. 2012; 35: 557-564. 